\title{
Synergistic effect and application of xylanases as accessory enzymes to enhance the hydrolysis of pretreated bagasse.
}

Geisa A. L. Gonçalves ${ }^{\mathrm{a}+}$, Yusaku Takasugi ${ }^{\mathrm{a}}$, Lili Jia ${ }^{\mathrm{a}}$, Yutaro Mori ${ }^{\mathrm{a}}$, Shuhei Noda ${ }^{\mathrm{b}}$, Tsutomu Tanaka ${ }^{\mathrm{c}}$, Hirofumi Ichinose ${ }^{\mathrm{d}}$, Noriho Kamiya ${ }^{\mathrm{a}, \mathrm{e}_{*}}$

${ }^{a}$ Department of Applied Chemistry, Graduate School of Engineering, Kyushu University, Japan.

${ }^{\mathrm{b}}$ Biomass Engineering Program, RIKEN, 1-7-22, Suehiro-cho, Tsurumi-ku, Yokohama, Kanagawa 230-0045, Japan.

${ }^{c}$ Department of Chemical Science and Engineering, Graduate School of Engineering, Kobe University, 1-1 Rokkodai, Nada, Kobe 657-8501, Japan.

${ }^{\mathrm{d}}$ Faculty of Agriculture, Kyushu University, Hakozaki 6-10-1, Higashi-ku, Fukuoka, 812-8581, Japan.

${ }^{\mathrm{e}}$ Center for Future Chemistry, Kyushu University, Japan.

${ }^{+}$These authors contributed equally to this work.

*Corresponding Author

a,e 744, Motooka, Nishi-Ku, Fukuoka, Japan

*nori_kamiya@mail.cstm.kyushu-u.ac.jp

Phone: (+81) 092-802-2807, Fax: (+81) 092-802-2810 


\section{$1 \quad$ Abstract}

2

Recently, the new trend in the second-generation ethanol industry is to use mild

3 pretreatments, in order to reduce costs and to keep higher content of hemicellulose in

4 the biomass. Nevertheless, a high enzyme dosage is still required in the conversion of

5 (hemi)cellulose. The interaction between cellulases and xylanases seems to be an

6 effective alternative to reduce enzyme loading in the saccharification process. At first,

7 to evaluate the synergism of xylanases on bagasse degradation, we have produced two

8 xylanases from glycoside hydrolase family 10 (GH10) and three xylanases from

9 glycoside hydrolase family 11 (GH11), from two thermophilic organisms, Thermobifida

10 fusca and Clostridium thermocellum, and one mesophilic organism, Streptomyces

11 lividans. Peracetic acid (PAA) pretreated bagasse was used as substrate. The

12 combination of XynZ-C (GH10, from C. thermocellum), and XlnB (GH11, from $S$.

13 lividans) presented the highest degree of synergy after 6 hours (3.62). However, the

14 combination of XynZ-C and Xyn11A (GH11, from T. fusca) resulted in the highest total

15 yield of reducing sugars. To evaluate the synergism between xylanases and cellulases,

16 commercial cellulase preparation from Trichoderma reesei was combined with the

17 selected xylanases, XynZ-C and Xyn11A. About 2-fold increase was observed in the

18 concentration of reducing sugars, when both xylanases, XynZ-C and Xyn11A, were

19 added together with $T$. reesei cellulases in the reaction mixture.

21 Keywords: Xylanase; Synergism; Lignocellulose; Bioconversion; Biofuel. 


\section{Introduction}

Xylanases have been traditionally used in several industrial processes over the

26 past decades, especially in the food, feed, detergents and pulp and paper segments.

27 However, xylanases are becoming more and more attractive for the saccharification

28 process of lignocellulosic biomass, once they can hydrolyze xylan and assist in the

29 hydrolysis of cellulose, to obtain fermentable sugars with potential use in the production

30 of biochemicals and biofuels [1]. Biofuel from cellulosic biomass is a major focus of

31 different governments and industries around the world. One of the bottlenecks in the

32 process of biofuel production is still the initial conversion of biomass into sugars, and

33 new biotechnological solutions are needed to make this process cost-competitive [2].

34 Several biomass conversion processes rely on biomass pretreatment before

35 enzymatic hydrolysis, to remove lignin and to reduce recalcitrance of the biopolymer

36 [3], but a reduction in the hemicellulose content can also occur. Recently, less severe

37 pretreatments of lignocellulosic biomass are gaining popularity, thus, hemicellulase

38 characterization and studies regarding synergism of cellulases and xylanases, can

39 contribute to reducing pretreatment severities and enhancing glucose and xylose release

40 [4]. The use of xylanases, as accessory enzymes, has shown to improve the hydrolysis

41 of xylan and cellulose, and it has contributed to the reduction of enzyme dosage, but it

42 seems to be a substrate dependent reaction [5-6].

43 Hemicellulolytic microorganisms usually produce multiple enzymes with

44 different mechanisms of action that can be combined to promote a unique synergism

45 during the saccharification process. Furthermore, these microorganisms usually co-exist

46 with other cellulolytic or xylanolytic organisms, creating an ideal ecosystem for plant 
47 cell wall degradation [7]. Mimicking the nature, diverse enzymes are being combined in selected formulas of cocktails to be applied in industrial process. A recent study has shown that most of the xylanases used in the industry are from mesophilic and/or neutrophilic origin, but thermophilic and acidophilic enzymes would be of interest in many biotechnological processes [8]. In fact, highly stable enzymes, active under extreme conditions, could bring many advantages for several biotechnological processes, as contamination reduction and faster reaction rates, and it could lead to a breakthrough in the development of (hemi)cellulases for biomass conversion [9]. There is a growing interest in xylanases from thermophilic organisms at the industrial level, because of their ability to degrade xylan at high temperatures [10]. Thermophilic enzymes have also been identified in the deconstruction of lignocellulose, and the characterization of these enzymes could represent new opportunities in the renewable biofuel area [11]. As a result, thermophilic cellulolytic organisms and their enzymes are gaining notoriety in the bioconversion field [12]. Exploring the use of novel enzymes and the combination of enzymes from different organisms, with distinct characteristics, could contribute to the development of a highly efficient system for lignocellulose conversion. pretreated bagasse, family 10 and 11 xylanases were produced. Overall, we observed that higher synergism occurred between the xylanases from different families (GH10 and GH11). Ultimately, the synergism between xylanases and cellulases were evaluated. The addition of the binary mixture of xylanases, XynZ-C and Xyn11A, has increased

69 the activity of the commercial cellulase mixture in the hydrolysis of bagasse. 


\section{Materials and Methods}

\subsection{Biomass material and enzymes}

The biomass, bagasse, particle size average of $200 \mu \mathrm{m}$, was purchased from

Toyota Tsusho Corporation (Nagoya, Japan). Commercial cellulase from Trichoderma reesei was purchased from Sigma-Aldrich. Other enzymes used in this study are indicated in Table 1, and they were expressed and purified as follows.

\section{$\mathrm{Z}(\mathrm{GH} 10)$}

\subsubsection{Expression and purification of xylanases from Clostridium thermocellum} Genomic DNA of Clostridium thermocellum (NBRC 103400) was obtained from NITE Biological Resource Center (Japan). A gene fragment encoding xylanase domain of XynZ (M22624) was amplified from the $C$. thermocellum genome using Phusion DNA polymerase (Thermo Fisher Scientific). The PCR mixture (50 $\mu \mathrm{L})$ contained 1 U Phusion High-Fidelity DNA Polymerase, $1 \times$ Phusion HF Buffer, 200 $\mu \mathrm{M}$ dNTPs, $10 \mathrm{ng}$ genomic DNA, and 100 pmol each of primers (Forward primer; 5'AAGGAGATATACATATGACTCCGACACAAACTCCTATCCCCA-3', Reverse primer; 5'-GGTGGTGGTGCTCGAGATAGCCCATAAGAGCTTCCTTTATTGC-3').

The gene fragment was amplified without the original stop codon to introduce a hexaHis-tag from an expression plasmid. PCR conditions were as follows: $94^{\circ} \mathrm{C}$ for $60 \mathrm{~s}$ followed by $94^{\circ} \mathrm{C}$ for $10 \mathrm{~s}, 60^{\circ} \mathrm{C}$ for $15 \mathrm{~s}$, and $72^{\circ} \mathrm{C}$ for $60 \mathrm{~s}$ for 35 cycles, and then a final 2 min extension at $72^{\circ} \mathrm{C}$. The amplified gene fragment was purified using a QIAquick Gel Extraction Kit (Qiagen, Tokyo, Japan) and cloned into NdeI/XhoI site of 
94 pET22 vector (Novagen, Madison, WI, USA) using In-Fusion ${ }^{\circledR}$ HD Cloning Kit

95 (Clontech Laboratories, Inc., Mountain View, CA, USA).

E. coli $\mathrm{C} 41$ (DE3) was transformed with the expression plasmid of xylanase. A

97 fresh transformant was seeded into $50 \mathrm{~mL}$ of Luria-Bertani medium supplemented with

98 carbenicillin $(50 \mathrm{mg} / \mathrm{L})$ and grown overnight with shaking $(180 \mathrm{rpm})$ at $37^{\circ} \mathrm{C}$. The

99 overnight culture was transferred into $1 \mathrm{~L}$ of Terrific Broth medium supplemented with

100 carbenicillin $(50 \mathrm{mg} / \mathrm{L})$. Bacterial cells were grown with shaking $(180 \mathrm{rpm})$ at $37^{\circ} \mathrm{C}$

101 until the $\mathrm{OD}_{600}$ reached 0.8 . The culture was then transferred to $27^{\circ} \mathrm{C}$, supplemented

102 with IPTG $(0.5 \mathrm{mM})$, and incubated with shaking (180 rpm) for $48 \mathrm{~h}$. After $48 \mathrm{~h}$

103 incubation, bacterial cells were harvested by centrifugation $(7,000 \mathrm{~g})$, washed with 100

$104 \mathrm{~mL}$ of $50 \mathrm{mM}$ potassium phosphate $(\mathrm{pH} 7.4)$, and then collected by centrifugation

$105(7,000 \mathrm{~g})$. The cells were re-supplemented in $40 \mathrm{~mL}$ lysis buffer consisting of $50 \mathrm{mM}$

106 HEPES (pH7.4), 1mM EDTA, 1mM DTT, 1mM PMSF, and 20\% glycerol, and then

107 disrupted by sonication in an ice-cold water bath. After removing cell debris by

108 centrifugation $(10,000 \mathrm{~g})$, the resultant cell-free extract was applied for His-tag affinity

109 chromatography using an AKTA Prime plus system (GE Healthcare) equipped with

110 HisTrap FF crude columns (GE Healthcare) at $4^{\circ} \mathrm{C}$. After the affinity chromatography,

111 recombinant protein was further purified by anion-exchange chromatography using

112 HiTrap Q HP column (GE Healthcare). After the chromatographic purification, the

113 recombinant protein was concentrated using a centrifugal filter device (Amicon Ultra-

114 15, Millipore) and exchanged into $50 \mathrm{mM}$ HEPES (pH 7.4) containing 20\% glycerol,

$1150.5 \mathrm{mM}$ DTT using a PD10 column (GE Healthcare).

116 
118 Xyn10B or Tfu2791 (GH10) and Xyn11A or Tfu1213 (GH11), and Streptomyces

119 lividans $-\mathrm{X} \operatorname{lnB}$ and $\mathrm{X} \operatorname{lnC}(\mathrm{GH} 11)$

120 Each polymerase chain reaction (PCR) was carried out using PrimeSTAR HS

121 (Takara, Shiga, Japan). The plasmids for expressing Tfu2791 (GH10) or Tfu1213

122 (GH11) were constructed as follows. The gene fragments encoding Tfu2791 (GH10) or

123 Tfu1213 (GH11) were amplified by PCR using the Thermobifida fusca YX genome

124 (ATCC number: BAA-629D-5) as a template with the following primers: 5'-

125 TCGTTtAAGGATGCAATGAAACCGGTGCGTCTCATCACCG-3’ and 5'-

126 CGCTCAGTCGTCTCATCAGTGGTGGTGGTGGTGGTGGCAGTGATCGTGCTTG

127 GGGC-3' or 5'-TCGTTTAAGGATGCAATGAACCATGCCCCCGCCAGTCTGA-3'

128 and 5

129

CGCTCAGTCGTCTCATCAGTGGTGGTGGTGGTGGTGGTTGGCGCTGCAGGAC

130 ACCG-3'. Each fragment was introduced into the SphI and BglII sites of pUC702-pro-

131 sig-term [13], using an In-Fusion HD Cloning Kit (Takara). The resultant plasmid was

132 called pUC702-Tfu2791 or pUC702-Tfu1213.

133 The plasmids for expressing $\mathrm{X} \operatorname{lnB}$ or $\mathrm{X} \operatorname{lnC}(\mathrm{GH} 11)$ were constructed as follows.

134 The gene fragments encoding $\mathrm{X} \operatorname{lnB}$ or $\mathrm{X} \operatorname{lnC}(\mathrm{GH} 11)$ were amplified by PCR using the

135 Streptomyces lividans 1326 (NBRC15675) as a template with the following primers: 5'-

136 TCGTTTAAGGATGCAATGAACCTGCTCGTCCAGCCGAGGC -3’ and 5’-

137 CGCTCAGTCGTCTCATCAGTGGTGGTGGTGGTGGTGGCCCGCGCTGCAGGA

138 CACGCTG -3 ' or 5'

139 TCGTTtAAGGATGCAATGCAGCAGGACGGCACACAGCAGG-3’' and 5'-

140 CGCTCAGTCGTCTCATCAGTGGTGGTGGTGGTGGTGACCGCTGACCGTGATG 
141 TTCGAG-3'. Each fragment was introduced into the SphI and BglII sites of pUC702-

142 pro-sig-term using an In-Fusion HD Cloning Kit. The resultant plasmid was called 143 pUC702-XlnB or pUC702-XlnC.

144 Protoplasts of wild-type S. lividans 1326 was prepared according to the method 145 of Hopwood et al. [14]. Briefly, the mycelium of each strain was treated with a solution 146 of $1 \mathrm{mg} / \mathrm{mL}$ lysozyme (Wako, Osaka, Japan), and suspended mycelia were used as 147 protoplasts. Each multi-copy plasmid (pUC702-Tfu2791, pUC702-Tfu1213, pUC702-

$148 \mathrm{X} \operatorname{lnB}$ or pUC702-XlnC) was introduced into wild-type S. lividans 1326 using the 149 polyethylene glycol (PEG) method. Selection of transformants was carried out by 150 overlaying soft agar containing $50 \mu \mathrm{g} / \mathrm{ml}$ of thiostrepton. After cultivation for 5 days, 151 transformants were selected and named as S. lividans/Tfu2791, S. lividans/Tfu1213, S. 152 lividans/XlnB, and S. lividans/XlnC.

153 Spore suspension of each transformant was inoculated in a test tube containing 5

$154 \mathrm{~mL}$ of tryptic soy broth (TSB) medium (BD Diagnostic Systems, 199 Sparks, MD,

155 USA) supplemented with $5 \mu \mathrm{g} / \mathrm{ml}$ of thiostrepton (MP Biomedicals, Illkirch-

156 Graffenstaden, France), followed by cultivation at $28{ }^{\circ} \mathrm{C}$ for 3 days. Then, $5 \mathrm{ml}$ of the 157 preculture broth of each transformant was seeded into a shake flask with a baffle 158 containing $100 \mathrm{ml}$ of modified TSB medium with $5 \mu \mathrm{g} / \mathrm{ml}$ thiostrepton, $3 \%$ glucose, 159 and $5 \%$ tryptone, followed by incubation at $28{ }^{\circ} \mathrm{C}$ for 4 days.

160 Each culture supernatant (300 ml) of S. lividans/Tfu2791, S. lividans/Tfu1213, S.

161 lividans/XlnB, or S. lividans/XlnC was precipitated by ammonium sulfate. The 162 precipitate of each was collected by centrifugation at 20,000g for $30 \mathrm{~min}$, and dissolved 163 with $50 \mathrm{mM}$ phosphate buffer (pH 7.0) containing $300 \mathrm{mM} \mathrm{NaCl}$. Tfu2791, Tfu1213, $164 \mathrm{X} \operatorname{lnB}$, and $\mathrm{X} \operatorname{lnC}$ were separately purified using TALON metal affinity resins (Takara) 
165 according to the manufacturer's protocol, and dialyzed with $50 \mathrm{mM}$ phosphate buffer 166 (pH 7.0) containing $300 \mathrm{mM} \mathrm{NaCl}$.

167

168 2.2. Enzyme specific activity

169 Xylanase specific activity was assayed using beechwood xylan (Tokyo Chemical 170 Industry Co., Ltd.). The beechwood xylan (0.25 w/v\%) was dissolved in $50 \mathrm{mM}$ 171 phosphate buffer, $\mathrm{pH} 7.0$, and xylanase was added to a final concentration of 2.5

$172 \mu \mathrm{g} / \mathrm{mL}$. The mixture was incubated at $50^{\circ} \mathrm{C}$ for $10 \mathrm{~min}$. The amount of reducing sugars 173 released was determined by the dinitrosalicylic acid (DNS) method [15].

174

175

176

177 PAA solution $40 \%$ (w/w) (Mitsubishi Gas Chemical Company, Inc., Japan). The

178 mixture was heated at $80{ }^{\circ} \mathrm{C}$ with stirring (200 rpm) for $3 \mathrm{~h}$. After that, $5 \mathrm{~mL}$ of

179 deionized water was added to the mixture, and the sample was centrifuged at $5800 \mathrm{~g}$ for

$18020 \mathrm{~min}$, supernatant was discharged after centrifugation. To remove residual acids, the

181 solids were washed, by addition of $5 \mathrm{~mL}$ of water, followed by centrifugation ( $5800 \mathrm{~g} /$

$18220 \mathrm{~min}$ ), for 3 times. The regenerated bagasse was dried, overnight, by freeze-drying.

183

184

2.4. Chemical composition of the untreated and the PAA-treated bagasse

185

The chemical composition of the untreated and the PAA-treated bagasse was

186 measured following the protocol (LAP TP-510-42619, 42618, 42622) from National

187 Renewable Energy Laboratory, [16],[17], [18]. Briefly, the samples were treated with

$18872 \%$ sulfuric acid at $30{ }^{\circ} \mathrm{C}$ for $1 \mathrm{~h}$, then diluted to $4 \%$ and incubated in autoclave at 
$189121^{\circ} \mathrm{C}$ for $1 \mathrm{~h}$. After cooling, calcium carbonate was used for neutralizing the $\mathrm{pH}$ to 7.

190 Analysis of sugar content was performed using a high performance liquid

191 chromatography (HPLC) system equipped with Shodex sugar KS-801 column $(8.0 \times 300$

192 mm, Showa Denko Co., Tokyo, Japan) and RI detector at $80^{\circ} \mathrm{C}$, with HPLC-grade

193 water as the eluent (flow rate of $1 \mathrm{~mL} / \mathrm{min}$ ). The remaining acid insoluble lignin was

194 measured after oven-dried at $45^{\circ} \mathrm{C}$ overnight, and the amount of acid soluble lignin was

195 determined by measuring the absorbance at $240 \mathrm{~nm}$, against a deionized water blank

196 using a UV-Vis spectrophotometer (Jasco UV-Vis spectrophotometer V-550).

197

198

199

200

201

202

203

204

205

206

207

208

209

210

211

212

\subsection{Enzymatic saccharification}

The enzymatic saccharification was tested in a $2 \mathrm{~mL}$ final volume containing pretreated bagasse $(0.25 \mathrm{wt} \%$ biomass $)$, in $50 \mathrm{mM}$ sodium phosphate buffer $\mathrm{pH} 7.0$ for xylanase tests and pH 6.0 for cellulase and xylanase tests. The preliminary tests using xylanase only, enzyme loading was kept at $1 \mathrm{mg}$ of protein / $\mathrm{g}$ of biomass, for total enzyme added in the reaction tube. A ratio of 50:50 was used, when xylanases were mixed. Further tests, with xylanase and cellulase, enzyme loading was kept at $2 \mathrm{mg}$ of protein / $\mathrm{g}$ of biomass, for total enzyme added in the tube. A ratio of 50:50, cellulase:xylanase, or 50:25:25, cellulase:xylanase1:xylanase2, was used even when xylanases were combined to the cellulase commercial mix. All reactions were carried out at $50{ }^{\circ} \mathrm{C}$ with stirring (1000 rpm), for 72 hours. The DNS assay was performed to measure the reducing ends of (hemi)cellulose after the enzymatic reaction [15]. At the end of the enzyme reaction, $100 \mu \mathrm{L}$ of DNS reagent containing 1.3 M DNS, $1 \mathrm{M}$ potassium sodium tartrate, and $0.4 \mathrm{~N} \mathrm{NaOH}$ was added to $100 \mu \mathrm{L}$ of the reaction mixture containing substrate and incubated at $99{ }^{\circ} \mathrm{C}$ for $5 \mathrm{~min}$ to label the reducing ends 
213 of the hydrolyzed cellulose. Samples were analyzed at hour 3, 6, 12, 24, 48 and 72.

Quantification of soluble sugars were conducted based on the derivatization of

217 carbohydrates with ABEE (4-aminobenzoic acid ethyl ester) [19]. Briefly, to determine

218 the concentration of xylose, xylobiose and xylotriose, enzymatic hydrolysis samples

219 were centrifuged at $5800 \mathrm{~g}$ for 3 minutes, and the aqueous supernatant was filtered $(0.20$

$220 \mu \mathrm{m})$, in order to completely remove bagasse content. After that, $10 \mu \mathrm{l}$ of filtered samples were diluted in $40 \mu \mathrm{l}$ of ABEE reagent containing $1.4 \mathrm{M}$ sodium cyanoborohydride, 2.6 M benzocaine, 22.1 M methanol, and 1.8 M glacial acetic acid, and incubated at $83{ }^{\circ} \mathrm{C}$ for 1 hour. After incubation, samples were cooled down to 15 ${ }^{\circ} \mathrm{C}, 200 \mu \mathrm{l}$ of water and $200 \mu \mathrm{l}$ of chloroform were added to the mix. Samples were centrifuged at $5800 \mathrm{~g}$ for 3 minutes, and the final supernatant was used for HPLC analysis on a Shimadzu HPLC system equipped with a Honenpak C18 column (J-Oil Mills Inc., Japan, Tokyo, Japan), and an UV detector. HPLC method was run at a constant flow rate of $1.0 \mathrm{~mL} / \mathrm{min}$ with, $10 \%$ of acetonitrile and $90 \%$ of $0.02 \%$ TFA, as

229 the mobile phase, at $40{ }^{\circ} \mathrm{C}$ for $30 \mathrm{~min}$ and the elution was monitored at $305 \mathrm{~nm}$.

230 Standard curves of xylose, xylobiose and xylotriose, were prepared to determine the elution time and the peak areas as a function of the sugar concentration, in order to identify all sugars present in each sample as well as their concentrations. Samples were analyzed only in the end of the reaction, at hour 72 . 

determined as following equation [20] :

$$
\mathrm{DS}_{\mathrm{XYL}}=\mathrm{Y}_{1+2} /\left(\alpha \mathrm{Y}_{1}+\beta \mathrm{Y}_{2}\right)
$$

240 the case of the binary mixture of xylanases, $\alpha$ and $\beta$, correspond to $0.5, \mathrm{Y}_{1+2}$, indicates

241 the yield of reducing sugar achieved by the two enzymes working simultaneously, $\mathrm{Y}_{1}$ and $\mathrm{Y}_{2}$ indicate the yields of reducing sugar, achieved by each enzyme working separately.

245 the two selected xylanases, the following equation was applied:

$$
\mathrm{DS}_{\mathrm{XYL}+\mathrm{CEL}}=\mathrm{Y}_{1+2+3} /\left(\alpha \mathrm{Y}_{1}+\beta \mathrm{Y}_{2}+\gamma \mathrm{Y}_{3}\right)
$$

247 Where $\alpha, \beta$ and $\gamma$ correspond to the mass ratio of the enzyme in each reaction. In the 248 case of the xylanases, $\mathrm{Y}_{1}$ and $\mathrm{Y}_{2}, \alpha$ and $\beta$, correspond to 0.25 , and for the commercial 249 cellulase $\mathrm{Y}_{3}, \gamma$ correspond to $0.5, \mathrm{Y}_{1+2+3}$, indicates the yield of reducing sugar achieved 250 by all enzymes working simultaneously, $\mathrm{Y}_{1}, \mathrm{Y}_{2}$ and $\mathrm{Y}_{3}$ indicate the yields of reducing 251 sugar achieved by each kind of enzyme working separately.

252 As a general rule, DS indicates the cooperation between the involved enzymes:

253 If DS is higher than one, there is cooperation among the involved enzymes.

254 If DS is lower than one, there is competition of the involved enzymes.

255 High values of DS indicate high cooperation among the involved enzymes. 
262 C), and S. lividans (XlnB and XlnC) were purified by affinity chromatography, as

263 described in materials and methods section, enzyme purities were confirmed by SDS-

264 PAGE (Figure 1). Specific activities were determined using beechwood xylan as

265 substrate (Table 1). The mesophilic xylanase XlnC (GH11) showed the highest activity

266 on beechwood xylan (119.5 U/mg), followed by the thermophilic, family 10, xylanase

267 XynZ-C (93.0 U/mg). Family 11, xylanase XlnB (60 U/mg), and the thermophilic

268 xylanases, family 11, Xyn11A (51 U/mg) and, family 10, Xyn10B (45.3 U/mg)

269 presented lower hydrolytic activity on beechwood xylan. Therefore, it was not possible

270 to conclude if xylanases from family 11 were more susceptible to have higher activity

271 on soluble beechwood xylan substrate than xylanases from family 10, or vice versa.

272 Previous studies have demonstrated distinct preferences between GH10 and GH11

273 xylanases to soluble xylan substrates [6], [12]. Thus, the affinity and hydrolytic activity

274 of a certain enzyme to a specific substrate seems to vary according to the enzyme

275 origin, type, structure, family, and other factors.

Pretreatment method is usually necessary, before saccharification process, to

277 increase the accessibility of hemicellulase and cellulase enzymes to woody biomass.

278 Aiming to remove maximum content of lignin, but at the same time, to keep maximum

279 content of cellulose and hemicellulose, PAA method was chosen to pretreat the bagasse.

280 The PAA treatment is a conventional method, well known and used in the pretreatment

281 of lignocellulosic biomass, especially in softwoods and hardwoods. Nevertheless, the

282 successful use of PAA on the pretreatment of agricultural residues, with high

283 delignification and less severe impacts was demonstrated [21]. As expected, cellulose 
content was not changed and xylan removal was minimum (4.5\%) in the PAA-treated bagasse. On the contrary, the removal of lignin was not as high as expected; only about $5.7 \%$ of lignin content was removed. This could be likely due to the low PAA loading ( $0.4 \mathrm{~g}$ of PAA/ $\mathrm{g}$ of biomass) used in the bagasse pretreatment. Even thought pretreatment temperature was $80^{\circ} \mathrm{C}$, reaction time was about 3 hours. Kumar and coworkers have demonstrated low lignin removal at low PAA loading (0.5 g of PAA/ $\mathrm{g}$ of biomass) at $25^{\circ} \mathrm{C}$ after 24 hours, but high lignin removal, at higher loading of PAA (5.5 $\mathrm{g}$ of PAA/ $\mathrm{g}$ of biomass) at $25^{\circ} \mathrm{C}$ after 48 hours [21]. Surprisingly, $68 \%$ of the arabinan content was removed from the original bagasse, likely favoring the action of the xylanases in the PAA-treated bagasse (Table 2). Arabinose is usually present in agricultural crops, combined with xylan. The inter- or intra-molecular crosslinks of arabinose and xylan could hinder the xylanase action during biomass degradation [22]. Previous study has demonstrated an inverse relationship between arabinose content and catalytic efficiency of a family 11 xylanase from Thermobacillus xylanilyticus [23].

3.2. Enzymatic saccharification of pretreated bagasse by xylanases from family 10 and family 11

The xylanolytic system of microbes to breakdown xylooligosaccharides is well

302 known and described in the literature. Microorganisms usually produce different types 303 of xylanases, which act in synergism to degrade plant cell wall [24]. Inspired by nature, 304 enzyme cocktails are being formulated to hydrolyze lignocellulosic biomass in 305 industrial processes. These cocktails of enzymes usually contain cellulases, and accessory enzymes, such as hemicellulases, from different families and/or microorganisms [25]. To investigate the synergism between distinct xylanases during 
enzymatic hydrolysis of bagasse, we have mixed xylanases from family 10 and family 11 (Table 1), in three modes: xylanases from family 10 mixture, xylanases from family

31011 mixture and xylanases from family 10 and family 11 mixture. Each individual 311 enzyme was also evaluated, in order to analyze the synergism between the different 312 combinations of enzymes. All enzyme mixtures were done in a ratio of 50:50 in this 313 study. Other ratios (10:90, 20:80, 30:70, 40:60) were also analyzed, however the best synergism was observed when mixing the xylanases in a ratio of 50:50 (data not shown).

Among all the tested enzymes, XynZ-C, from C. thermocellum, and Xyn11A,

317 from $T$. fusca, produced the highest concentration of reducing sugars, when tested sole

318 (Figure 2). Normally, Clostridium species are specialized in crystalline cellulose 319 degradation, but this organism produces several hemicellulases, especially xylanases 320 [26]. Early studies, demonstrated that pure xylanase Z, from C. thermocellum, was 321 shown to have high activity on xylan, but in nature $\mathrm{XynZ}$ is found to be associated with 322 the cellulosome structure [27]. More recently, XynZ, purified from C. thermocellum 323 cellulosome, was found to consist of a multidomain structure, with a N-terminus 324 ferulolyl esterase domain, a family 6 carbohydrate binding domain, a dockerin and a 325 glycoside hydrolase family 10 domain [28]. Sajjad and co-workers have previously 326 demonstrated higher activity of XynZ without the native non-catalytic domains before 327 in birchwood xylan [29]. The role of CBM was also analyzed by the attachment of 328 CBM6 to the C-terminal or N-terminal, and CBM22 in the N-terminal, of the catalytic 329 domain of XynZ, without the ferulolyl domain. The activity of XynZ was improved 330 only by the addition of CBM22 in the N-terminal of the catalytic domain. Nonetheless, 331 the specific activity of each enzyme form was demonstrated only on soluble xylan 
332 substrate [30]. In this study, we have expressed and purified the catalytic domain of

333 XynZ only, without ferulolyl esterase and CBM. Although, CBM is known to be

334 essential for the proximity of the enzyme and the substrate, high saccharification

335 efficiency was obtained by XynZ-C lacking CBM. Further work would be necessary to

336 analyze the impact of CBM addition to XynZ-C on the hydrolysis of bagasse.

337 The xylanase Xyn11A, from T. fusca, was shown to have a special binding

338 module that can bind strongly to both cellulose and xylan. Although Xyn11A has no

339 activity in cellulose, this special feature can potentially increase hemicellulose

340 degradation [31]. Recent study has demonstrated that a family 11 xylanase was more

341 efficient in hydrolyzing xylan, than a family 10 xylanase, in different soluble substrates

342 [6]. In addition, a GH11 xylanase from Thermobacillus xylanilyticus, had better

343 hydrolytic activity in wheat bran than a GH10 xylanase from the same organism [23].

344 However, the enzyme action seems to be directly related to the substrate source. For

345 example, the thermostable xylanase belonging to family 10 (Ta Xyn) was shown to be

346 more efficient in solubilizing xylan, from birchwood glucuronoxylan and wheat straw,

347 than a family 11 thermostable xylanase (Nf Xyn) [32]. Moreover, Kim and co-workers

348 have demonstrated that the Xyn10B, from T. fusca, has specific activity on various

349 substrates, and it produced more reducing sugars from corn fiber than the Xyn11A, for

350 example [33]. In the case of PAA pretreated bagasse, the family 10 xylanase, from $C$.

351 thermocellum, produced higher amounts of reducing sugars than both family 11

352 xylanases, from S. lividans, and similar amounts of reducing sugars when compared to

353 the family 11 xylanase, from $T$. fusca. On the other hand, the family 10 xylanase, from

354 T. fusca, yielded minimal amounts of reducing sugars during the hydrolysis of the 
pretreated bagasse. In addition to the substrate, it is likely that enzymatic action during the degradation of hemicellulose is related to the enzyme-producing organism.

family 10 and family 11 , from different or same organisms, for all tested combinations.

In contrast, minimum synergism was verified when mixing the xylanases from the same

360 family, GH10 or GH11. Previous report has shown synergistic effect between Xyn11A and Xyn10B from T. fusca, during hydrolysis of corn fiber [33]. The synergistic effect between enzymes from two different families could be expected due to the complex structure of natural hemicellulose, such as bagasse, that would require a dynamic enzyme action to be hydrolyzed. Xylanases belonging to family 10 usually have small binding sites and are highly active on short xylo-oligossaccharides, and may also be active on cellulose substrate. On the other hand, family 11 xylanases are exclusively active on xylose containing substrates. In addition, it has been demonstrated that hydrolytic products from family 11 xylanases can be further hydrolyzed by family 10 xylanases [34]. Unlike our data, synergistic effect was not observed when mixing GH10 and GH11 xylanases from the thermophilic bacterium Thermobacillus xylanilyticus, on the hydrolysis of wheat bran arabinoxylans [23]. Despite the fact that minimum synergism was observed when mixing xylanases from the same family (10 or 11) during

373 the hydrolysis of PAA-treated bagasse, other studies have shown synergism among

374 xylanases from family 11 only, from distinct organisms, in the bleaching of bagasse 375 pulp [35], or family 10 xylanases only, on hardwood and softwood paper pulps [36].

376 Taking into consideration the above-mentioned findings, it is clear that enzyme action is 377 highly dependent on the nature of substrate, origin of enzyme-producing microorganism 378 and enzymatic reaction conditions. 
3.3. Soluble sugar analysis of bagasse after enzymatic hydrolysis using different combinations of GH10 and GH11 Commercial development of hemicellulases is less advanced than cellulases in

383 the bioconversion field, especially because most of the biomass pretreatment still 384 depends on acid preparation, removing the majority of the hemicellulose component.

Nevertheless, studies involving hemicellulases have increased due to recent trends in reducing pretreatment severities to preserve hemicellulose content [4],[25]. Thus, there is a need to understand different aspects of plant biomass degradation. In order to evaluate and quantify the final content of soluble sugars on hydrolyzed bagasse, samples were analyzed by HPLC after 72 hours. Soluble sugar analysis has confirmed the results obtained in the quantification of reducing sugars, affirming that synergistic effect occurs when mixing xylanases from family 10 and family 11 (Figure 3). As expected, the xylanase Xyn10B, from $T$. fusca, generated shorter products than xylanases from family 11 , basically only xylose and xylobiose. Although xylanase 394 XynZ-C, from C. thermocellum, has produced the highest amount of xylose during 395 hydrolysis of the bagasse, this enzyme has also generated small amounts of xylotriose. 396 This could be explained by the differences in the active sites and/or substrate affinity, 397 once XynZ-C and Xyn10B originated from different organisms. Interestingly, the 398 xylanases from S. lividans (XlnB and XlnC), belonging to family 11, produced only 399 xylobiose and xylotriose when tested sole. However, the same enzymes when mixed 400 with Xyn10B, generated a mix of xylose and xylobiose, and no xylotriose was observed. Small amounts of xylose were also observed when mixing XlnB or XlnC with 402 Xyn11A, although these enzymes belong to the same family (GH11). Nonetheless, 
403 xylotriose was still produced in the reaction mix. In general, all reaction mixtures have 404 increased the final amount of xylose and xylobiose, while reducing total xylotriose.

405 Recent work has indicated that family 10 xylanases usually generate smaller products 406 than family 11 xylanases, but this tendency can vary according to substrate type and 407 enzyme-producing microorganism [8]. Amongst all the enzymes tested in this study, 408 XynZ-C generated the highest amount of xylose and xylobiose, when tested individually. Xyn11A has produced similar amounts of reducing sugars compared to XynZ-C, but less soluble sugars. Nevertheless, long soluble xylooligomers, such as

411 xylotetraose and xylopentose have not been measured here. In general, xylobiose and 412 xylotriose are the main xylooligomers produced by xylanases during the hydrolysis of 413 lignicellulosic biomass, however previous works have demonstrated the production of high chain xylooligomers (X6, X7, X8, X9, X10 and X11) by GH10 or GH11 xylanases, during the hydrolysis of birchwood and wheat arabinoxylan [8], [37]. different enzymes is the calculation of the degree of synergy (DS). DS can be calculated

421 based on the ratio of the yield of sugars released by enzymes working simultaneously to 422 the sum of the yield of sugars released by each enzyme separately, once they were 423 applied in the mixture in equal quantities [38]. There are several proposed models for enzymatic hydrolysis of cellulose, (for a review, see Zhang and Lynd, 2004 [39]). Here,

425 we have used a type of semi-mechanistic method with respect to the enzyme to 426 calculate the degree of synergy, using an equation already described and used in prior 
studies [20], [40]. Enzymatic hydrolysis based on reducing sugar quantification was used for calculus purpose. Overall, higher degree of synergy was observed when mixing xylanases from different $\mathrm{GH}$ families, comparing to xylanase mixtures of the same

430 family. At hour 6, DS was higher than one, for all the binary mixtures, between GH10 431 and GH11, indicating that the enzymes worked in cooperation to enhance hemicellulose 432 hydrolysis. In contrast, the mixtures between xylanases of the same family, such as, 433 XynZ-C and Xyn10B, and Xyn11A and XlnB, presented DS lower than one, indicating 434 a competition of the involved enzymes (Figure 4). Nevertheless, DS was higher than 435 one at hour 72, for all enzyme combinations (Table 3). The highest DS (3.62) was 436 observed at hour 6, when mixing XynZ-C and XlnB, but the highest final DS obtained 437 at hour 72, was observed when mixing Xyn10B and Xyn11A (2.16) or Xyn10B and $438 \mathrm{X} \operatorname{lnB}(2.15)$. In this specific case, DS indicates the cooperation between the combined enzymes, to enhance the hydrolysis of hemicellulose. However, the degree of synergy

440 between 2 hemicellulases is not always an indicative of the overall efficiency of

441 hemicellulose degradation. The highest obtained DS did not correspond to the highest 442 yield of reducing sugars in this work. A decrease of DS was observed with the increase 443 of the reaction time, for most of the tested binary mixtures. Nevertheless, a contrasting 444 difference was verified when changing the species of family 10 xylanase in the reaction 445 mix. Less reduction in the final value of DS was observed when using Xyn10B from $T$. 446 fusca, instead of XynZ-C from C. thermocellum (Table 3). Several experiments using 447 xylanases to hydrolyze lignocellulose biomass have shown that DS value decrease with 448 the extension of enzymatic hydrolysis. This decrease on DS appears to be associated 449 with the substrate modification during hydrolysis, such as crystallinity index, and/or 450 irreversible adsorption of enzymes by regions containing lignin [29-30]. 

a commercial cellulase and GH11, we have tested the combination of the two top xylanases in this study, 456 XynZ-C and Xyn11A, and the commercial cellulase from T. reesei. The optimum $\mathrm{pH}$ 457 for T. reesei cellulase is known to be 5, while the optimum $\mathrm{pH}$ of both xylanases, 458 XynZ-C and Xyn11A, is about 7, but here the combinations of these enzymes were analyzed at $\mathrm{pH}$ 6. Considering, that enzymes from different microorganisms can act

460 synergistically, $\mathrm{pH}$ adjustment offers the possibility of developing enzyme mixtures with higher activity than each enzyme in the original crude extract. Previous studies have already used $\mathrm{pH} 6$, as a compromise between the optimal $\mathrm{pH}$ for $T$. reese $i$ and $T$. fusca enzymes [41], [42].

Family 10 xylanase, XynZ-C, showed higher synergism than family11,

465 Xyn11A, when combined with T. reesei commercial cellulase. However, the use of 466 GH10 and GH11 xylanases, simultaneasly, promoted the highest improvement in the 467 hydrolytic activity of the commercial cellulase, at same enzyme loading (Figure 5). The 468 synergistic interaction between XynZ-C and Xyn11A, proved to be efficient when combined to commercial cellulase mixture in the enhancement of pretreated bagasse

470 hydrolysis. The development of new formulations of enzyme cocktails is being 471 approached by different research groups, to reduce enzyme loading for biomass 472 conversion. In addition, hemicellulase activity in commercial cellulase cocktails is 473 usually minimum and not enough to convert all hemicellulose content, especially from 474 biomass pretreated under milder conditions [43]. The addition of family 10 and 11 
475 xylanases to commercial cellulase preparations seems to be of high relevance for 476 industrial process. The interactions of xylan and cellulose in pretreated biomass reduce

477 the accessibility of cellulase to celulose structure, and the synergy factor for glucose

478 formation and reducing sugar release were shown to increase linearly with the xylan 479 content in the substrate [44]. Recent study has demonstrated that the combination of 480 xylanases (GH10 and GH11) and xyloglucanase (GH5), with commercial cellulase 481 mixture improved the overall cellulose hydrolysis in pretreated corn fiber, sweet 482 sorghum bagasse, corn stover, poplar and lodgepole pine, but the relative improvement seems to be substrate dependent [6]. Another recent study using sugarcane bagasse has

484 shown that the interaction between cellulases and xylanases can also be related to the 485 pretreatment method, and consequently chemical composition of the pretreated biomass 486 [45] .

Here, pure xylanases, were used as accessory enzymes, and clear synergism was observed between pure xylanases and the commercial cellulase preparation.

489 Interestingly, continuing synergy was observed between the commercial cellulase from 490 T. reesei and the binary xylanase mixture (XynZ-C and Xyn11A) (Figure 4), with a DS 491 reduction of $30 \%$, from hour 6 to hour 72 , compared to a reduction of $50 \%$ when the same binary mixture was applied sole to the pretreated bagasse (Table 4). These results 493 demonstrate the potential use of xylanases as accessory enzymes to reduce total enzyme 494 loading during the hydrolysis of pretreated bagasse. Further studies should be 495 performed in order to optimize an ideal ratio between xylanases and cellulases for minimum enzyme dosage and maximum conversion of the pretreated biomass. 

and 11, thus a comprehension of the interaction between these xylanases and cellulases

501 would be of the utmost importance for industrial application. In this study, we have first 502 evaluated the interaction among xylanases from GH10 and GH11 from three different 503 organisms, and we have identified that mixing the different GH families generates 504 significant synergistic effect during pretreated bagasse hydrolysis. Furthermore, the 505 synergism observed between GH10 and GH11 xylanases had positive effect on the hydrolytic activity of a commercial cellulase mixture from $T$. reesei during pretreated 507 bagasse hydrolysis.

\section{Acknowledgments} This research was supported by the Advanced Low Carbon Technology Research and Development Program (ALCA) from the Japan Science and Technology Agency

512 (JST). L. Jia thanks the China Scholarship Council (grant number: 201306300044). The authors declare that they have no competing interests.

\section{Author's contribution}


[1] Beg QK, Kapoor M, Mahajan L, Hoondal GS. Microbial xylanases and their industrial applications: a review. Appl Microbiol Biotechnol 2001;56:326-38.

[2] Lynd L, Laser M, Bransby D, Dale B. How biotech can transform biofuels. Nat

[3] Himmel ME, Ding S-Y, Johnson DK, Adney WS, Nimlos MR, Brady JW, et al.

[4] Harris P V, Xu F, Kreel NE, Kang C, Fukuyama S. New enzyme insights drive Science 2007;315:804-7.

advances in commercial ethanol production. Curr Opin Chem Biol 2014;19C:162-70.

[5] Hu J, Arantes V, Saddler JN. The enhancement of enzymatic hydrolysis of lignocellulosic substrates by the addition of accessory enzymes such as xylanase: is it an additive or synergistic effect? Biotechnol Bioeng 2011;36:1 -13. [6] Hu J, Arantes V, Pribowo A, Saddler JN. The synergistic action of accessory
enzymes enhances the hydrolytic potential of a "cellulase mixture" but is highly substrate specific. Biotechnol Biofuels 2013;6:112.

[7] Bayer E A, Morag E, Lamed R. The cellulosome--a treasure-trove for biotechnology. Trends Biotechnol 1994;12:379-86.

[8] Sydenham R, Zheng Y, Riemens A, Tsang A, Powlowski J, Storms R. Cloning and enzymatic characterization of four thermostable fungal endo-1,4- $\beta$ xylanases. Appl Microbiol Biotechnol 2014;98:3613-28.

[9] Kulkarni N, Shendye a, Rao M. Molecular and biotechnological aspects of xylanases. FEMS Microbiol Rev 1999;23:411-56.

[10] Cannio R, Di Prizito N, Rossi M, Morana A. A xylan-degrading strain of Sulfolobus solfataricus: isolation and characterization of the xylanase activity. Extremophiles 2004;8:117-24.

[11] Blumer-Schuette SE, Kataeva I, Westpheling J, Adams MW, Kelly RM.

Extremely thermophilic microorganisms for biomass conversion: status and prospects. Curr Opin Biotechnol 2008;19:210-7.

[12] Zhang J, Siika-Aho M, Puranen T, Tang M, Tenkanen M, Viikari L. Thermostable recombinant xylanases from Nonomuraea flexuosa and Thermoascus aurantiacus show distinct properties in the hydrolysis of xylans and pretreated wheat straw. Biotechnol Biofuels 2011;4:1-12. 
[13] Noda S, Ito Y, Shimizu N, Tanaka T, Ogino C, Kondo A. Over-production of various secretory-form proteins in Streptomyces lividans. Protein Expr Purif 2010;73:198-202.

[14] Hopwood DA. Genetic manipulation of Streptomyces: a laboratory manual. 1995.

[15] Miller GL. Use of dinitrosalicyclic reagent for determination of reducing sugar. Anal Chem 1959;31:426- 428.

[16] Sluiter A, Ruiz RO, Scarlata C, Sluiter J, Templeton D, Energy D of. Determination of Extractives in Biomass. 2004.

[17] Sluiter A, Hames B, Ruiz RO, Scarlata C, Sluiter J, Templeton D, et al. Determination of Structural Carbohydrates and Lignin in Biomass. Biomass Anal Technol Team Lab Anal Proced 2004;2011:1-14.

[18] Sluiter A, Hames B, Ruiz RO, Scarlata C, Sluiter J, Templeton D, et al. Determination of Ash in Biomass. 2004.

[19] Yasuno S, Murata T, Kokubi K, Yamaguchi T, Kamei M. Two-mode analysis by high-performance liquid chromatography of p-aminobenzoic ethyl esterderivatized monosaccharides. Biosci Biotechnol Biochem 1997:1944 - 1946.

[20] Andersen N, Johansen KS, Michelsen M, Stenby EH, Krogh KBRM, Olsson L. Hydrolysis of cellulose using mono-component enzymes shows synergy during hydrolysis of phosphoric acid swollen cellulose (PASC), but competition on Avicel. Enzyme Microb Technol 2008;42:362-70.

[21] Kumar R, Hu F, Hubbell C a, Ragauskas AJ, Wyman CE. Comparison of laboratory delignification methods, their selectivity, and impacts on physiochemical characteristics of cellulosic biomass. Bioresour Technol 2013;130:372-81.

[22] Gírio FM, Fonseca C, Carvalheiro F, Duarte LC, Marques S, Bogel-Łukasik R. Hemicelluloses for fuel ethanol: A review. Bioresour Technol 2010;101:4775800.

[23] Beaugrand J, Chambat G, Wong VWK, Goubet F, Rémond C, Paës G, et al. Impact and efficiency of GH10 and GH11 thermostable endoxylanases on wheat bran and alkali-extractable arabinoxylans. Carbohydr Res 2004;339:2529-40. doi:10.1016/j.carres.2004.08.012.

[24] Biely P. Microbial xylanolytic systems. Trends Biotechnol 1985;3:286-90.

[25] Mohanram S, Amat D, Choudhary J, Arora A, Nain L. Novel perspectives for evolving enzyme cocktails for lignocellulose hydrolysis in biorefineries. Sustain Chem Process 2013;1:1 - 12. 
602

603

604

605

606

607

608

609

610

611

612

613

614

615

616

617

618

619

620

621

622

623

624

625

626
[26] Zverlov V V, Schantz N, Schmitt-Kopplin P, Schwarz WH. Two new major subunits in the cellulosome of Clostridium thermocellum: xyloglucanase Xgh74A and endoxylanase Xyn10D. Microbiology 2005;151:3395-401.

[27] Grépinet O, Chebrou M, Béguin P. Purification of Clostridium thermocellum xylanase $\mathrm{Z}$ expressed in Escherichia coli and identification of the corresponding product in the culture medium of $C$. thermocellum. J Bacteriol 1988;170:4576 4581 .

[28] Blum DL, Kataeva IA, Li X, Lars G. Feruloyl Esterase Activity of the Clostridium thermocellum Cellulosome Can Be Attributed to Previously Unknown Domains of XynY and XynZ. J Bacteriol 2000;182:1346 - 1351.

[29] Sajjad M, Khan MIM, Akbar NS, Ahmad S, Ali I, Akhtar MW. Enhanced expression and activity yields of Clostridium thermocellum xylanases without non-catalytic domains. J Biotechnol 2010;145:38-42.

[30] Khan MIM, Sajjad M, Sadaf S, Zafar R, Niazi UHK, Akhtar MW. The nature of the carbohydrate binding module determines the catalytic efficiency of xylanase $\mathrm{Z}$ of Clostridium thermocellum. J Biotechnol 2013;168:403-8.

[31] Irwin D, Jung ED, Wilson DB. Characterization and sequence of a Thermomonospora fusca xylanase. Appl Environ Microbiol 1994;60:763-70.

[32] Zhang J, Tuomainen P, Siika-Aho M, Viikari L. Comparison of the synergistic action of two thermostable xylanases from GH families 10 and 11 with thermostable cellulases in lignocellulose hydrolysis. Bioresour Technol 2011;102:9090-5.

[33] Kim J, Irwin D, Wilson D. Purification and characterization of Thermobifida fusca xylanase 10B. Can J ... 2004;50:835-43.

[34] Collins T, Gerday C, Feller G. Xylanases, xylanase families and extremophilic xylanases. FEMS Microbiol Rev 2005;29:3-23.

[35] Bissoon, S., Singh, S. \& Christov L. Synergistic interactions of purified xylanases on bagasse pulp hemicellulose. African pulp Pap. week, 2002.

[36] Clarke JH, Rixon JE, Ciruela a, Gilbert HJ, Hazlewood GP. Family-10 and family-11 xylanases differ in their capacity to enhance the bleachability of hardwood and softwood paper pulps. Appl Microbiol Biotechnol 1997;48:17783.

[37] Zhang J, Viikari L. Xylo-oligosaccharides are competitive inhibitors of cellobiohydrolase I from Thermoascus aurantiacus. Bioresour Technol 2012;117:286-91. 
639

640

641

642

643

644

645

646

647

648

649

650
[38] Van Dyk JS, Pletschke BI. A review of lignocellulose bioconversion using enzymatic hydrolysis and synergistic cooperation between enzymes-Factors affecting enzymes, conversion and synergy. Biotechnol Adv 2012;30:1458-80.

[39] Zhang Y-HP, Lynd LR. Toward an aggregated understanding of enzymatic hydrolysis of cellulose: noncomplexed cellulase systems. Biotechnol Bioeng 2004;88:797-824.

[40] Fox JM, Jess P, Jambusaria RB, Moo GM, Liphardt J, Clark DS, et al. A singlemolecule analysis reveals morphological targets for cellulase synergy. Nat Chem Biol 2013;9:356-61.

[41] Bothwell MK, Walker LP, Prices M, Engineering B, Hall R, Biology C. SYNERGISM BETWEEN PURE THERMOMONOSPORA FUSCA AND TRICHODERMA. Biomass and Bioenergy 1993;4:293-9.

[42] Walker LP, Mcquire DWDCIC, Price M. Fragmentation of Cellulose by the Major Thermomonospora fusca Cellulases, Trichoderma reesei $\mathrm{CBHI}$, and Their Mixtures. Biotechnol Bioeng 1992;40:1019 - 1026.

[43] Berlin A, Maximenko V, Gilkes N, Saddler J. Optimization of Enzyme Complexes for Lignocellulose Hydrolysis. Biotechnol Bioeng 2007;97:287-96.

[44] Zhang J, Viikari L. Impact of xylan on synergistic effects of xylanases and cellulases in enzymatic hydrolysis of lignocelluloses. Appl Biochem Biotechnol 2014;174:1393-402.

[45] Li J, Zhou P, Liu H, Xiong C, Lin J, Xiao W, et al. Synergism of cellulase, xylanase, and pectinase on hydrolyzing sugarcane bagasse resulting from different pretreatment technologies. Bioresour Technol 2014;155:258-65. 
654 Figure 1. SDS-PAGE of the purified enzymes used in this study: XynZ-C (lane1), 655 Xyn11A (lane 2), Xyn10B (lane 3), XlnB (lane 4), XlnC (lane5) and marker (lane M). 656

657 Figure 2. Comparative hydrolysis of pretreated bagasse by xylanases from family 658 GH10, sole or mixed, and GH11, sole or mixed, from C. thermocellum, T. fusca and S. 659 lividans. Reactions were prepared by mixing enzymes from the same family (GH10 or 660 GH11), or from both families (GH10 and GH11). Enzymatic activity was measured as $661 \mathrm{mM}$ of reducing sugars. Reactions were performed for 72 hours, and samples were 662 taken at hour 3 (yellow bars), hour 6 (red bars), hour 12 (green bars), hour 24 (purple 663 bars), hour 48 (blue bars) and hour 72 (orange bars). Error bars are shown from 664 duplicates.

665

666 Figure 3. Determination of soluble sugars after hydrolysis of pretreated bagasse by 667 xylanases from family GH10, sole or mixed, and GH11, sole or mixed, from $C$. 668 thermocellum, T. fusca and S. lividans. Reactions were prepared by mixing enzymes 669 from the same family (GH10 or GH11), or from both families (GH10 and GH11). The 670 concentration of xylose (black bars), xylobiose (grey bars) and xylotriose (white bars)

671 were determined as $\mathrm{mM}$ after 72 hours of reaction. Error bars are shown from 672 duplicates.

673

674 Figure 4. Synergistic action of xylanases from family 10 and 11 during hydrolysis of 675 pretreated bagasse. Synergies were calculated at hour 6, for mixing enzymes of different 676 families, GH10 and GH11 (black), or same family, GH10 or GH11 (white). 
678 Figure 5. Comparative hydrolysis of pretreated bagasse by commercial cellulase

679 preparation from $T$. reesei (Cel), sole or mixed, with GH10 xylanase (XynZ-C) and/or

680 GH11 xylanase (Xyn11A). Enzymatic activity was measured as mM of reducing sugars.

681 Reactions were performed for 72 hours. Error bars are shown from duplicates.

682

683 
685 Table 1. Enzymes used in this study

686

\begin{tabular}{|c|c|c|c|c|c|}
\hline $\begin{array}{c}\text { Protein } \\
\text { name }\end{array}$ & CBM & Microorganism & $\begin{array}{c}\text { Xylanase } \\
\text { activity } \\
\text { (U/mg of } \\
\text { enzyme) }\end{array}$ & $\begin{array}{c}\text { MW } \\
\text { (kDa) }\end{array}$ & Family \\
\hline $\begin{array}{c}\text { XynZ-C } \\
-{ }^{\mathrm{a}}\end{array}$ & $\begin{array}{c}\text { Clostridium } \\
\text { thermocellum }\end{array}$ & 93.0 & 38.0 & GH10 \\
\hline $\begin{array}{c}\text { Xyn10B } \\
\text { (or } \\
\text { Tfu2791) }\end{array}$ & $-{ }^{\mathrm{b}}$ & Thermobifida fusca & 45.3 & 45.1 & GH10 \\
\hline $\begin{array}{c}\text { Xyn11A } \\
\text { (or } \\
\text { Tfu1213) }\end{array}$ & XBM $^{\mathrm{c}}$ & Thermobifida fusca & 51.0 & 36.4 & GH11 \\
\hline XlnB & $\mathrm{CBM}^{\text {Xnd }}$ & Streptomyces lividans & 60.0 & 35.6 & GH11 \\
\hline XlnC & $-{ }^{\mathrm{b}}$ & Streptomyces lividans & 119.5 & 24.7 & GH11 \\
\hline
\end{tabular}

687

$688{ }^{a}$ XynZ consists of N-terminus ferulolyl esterase domain, a family 6 carbohydrate 689 binding domain, a dockerin and a glycoside hydrolase family 10 domain. However, in 690 this study, only the catalytic domain was expressed (XynZ-C).

$691{ }^{b}$ The specific enzymes do not contain CBM.

$692{ }^{\mathrm{c}}$ XBM can bind to xylan or cellulose.

$693{ }^{\mathrm{d}} 1$ unit $(\mathrm{U})=1 \mu \mathrm{mol}$ of reducing sugars / $\mathrm{min}$

694 
696 Table 2. Chemical composition of the untreated and PAA-treated bagasse

\begin{tabular}{|c|c|c|}
\hline & Untreated & PAA pretreated \\
\hline Glucan (\%) & $37.7 \pm 1.1$ & $38.6 \pm 0.4$ \\
\hline Xylan (\%) & $22.0 \pm 5.6$ & $21.0 \pm 0.7$ \\
\hline Arabinan (\%) & $2.8 \pm 0.8$ & $0.9 \pm 0.3$ \\
\hline Ash (\%) & $3.7 \pm 0.4$ & $2.7 \pm 0.5$ \\
\hline Extractives (\%) & $13.1 \pm 1.6$ & $11.5 \pm 0.4$ \\
\hline Acid insoluble lignin (\%) & $20.2 \pm 0.8$ & $14.9 \pm 2.5$ \\
\hline Acid soluble lignin (\%) & $2.3 \pm 0.1$ & $6.3 \pm 0.3$ \\
\hline
\end{tabular}

709 
711 Table 3 - The degree of synergy of xylanases from glycoside hydrolase $(\mathrm{GH})$ families $712 \quad 10$ and 11.

713

\begin{tabular}{|c|c|c|c|c|c|c|c|c|c|c|}
\hline \multirow[b]{2}{*}{$\begin{array}{l}\text { Reaction } \\
\text { time (h) }\end{array}$} & \multicolumn{4}{|c|}{ Same GH family } & \multicolumn{6}{|c|}{ Different GH families } \\
\hline & $\begin{array}{c}\text { XynZ-C } \\
+ \\
\text { Xyn10B }\end{array}$ & $\begin{array}{c}\text { Xyn11 } \\
\text { A + } \\
\text { XlnB }\end{array}$ & $\begin{array}{c}\text { Xyn11A } \\
+ \\
\text { XlnC }\end{array}$ & $\begin{array}{c}\mathrm{X} \ln B \\
+ \\
\mathrm{X} \ln \mathrm{C}\end{array}$ & $\begin{array}{c}\text { XynZ-C } \\
+ \\
\text { Xyn11A }\end{array}$ & $\begin{array}{c}\text { XynZ-C } \\
+ \\
\text { XInB }\end{array}$ & $\begin{array}{c}\text { XynZ-C } \\
+ \\
\text { XInC }\end{array}$ & $\begin{array}{c}\text { Xyn10B } \\
+ \\
\text { Xyn11A }\end{array}$ & $\begin{array}{c}\text { Xyn10B } \\
+ \\
\text { XInB }\end{array}$ & $\begin{array}{c}\text { Xyn10B } \\
+ \\
\text { XInC }\end{array}$ \\
\hline 6 & 0.27 & 0.95 & 1.14 & 1.19 & 2.83 & 3.62 & 3.30 & 3.25 & 1.41 & 3.25 \\
\hline 12 & 0.98 & 0.99 & 0.85 & 1.51 & 1.89 & 3.58 & 2.28 & 2.44 & 2.93 & 2.14 \\
\hline 24 & 1.38 & 1.22 & 0.99 & 1.46 & 1.80 & 2.72 & 2.14 & 2.58 & 2.59 & 2.75 \\
\hline 48 & 1.47 & 1.22 & 1.08 & 1.30 & 1.46 & 2.22 & 1.73 & 2.23 & 2.30 & 2.02 \\
\hline 72 & 1.43 & 1.31 & 1.12 & 1.35 & 1.50 & 1.86 & 1.72 & 2.16 & 2.15 & 1.97 \\
\hline
\end{tabular}

714

715

716 
718 Table 4. The degree of synergy of xylanases and cellulases

719

\begin{tabular}{|l|l|l|l|l|}
\hline $\begin{array}{l}\text { Reaction } \\
\text { time (h) }\end{array}$ & $\begin{array}{l}\text { Cel + } \\
\text { XynZ-C }\end{array}$ & $\begin{array}{l}\text { Cel + } \\
\text { Xyn11A }\end{array}$ & $\begin{array}{l}\text { Cel + XynZ-C } \\
+ \text { Xyn11A }\end{array}$ & $\begin{array}{l}\text { XynZ-C + } \\
\text { Xyn11A }\end{array}$ \\
\hline 6 & 2.39 & 2.19 & 3.67 & 2.83 \\
\hline 12 & 2.41 & 1.91 & 3.19 & 1.89 \\
\hline 24 & 2.34 & 1.89 & 3.01 & 1.80 \\
\hline 48 & 2.26 & 1.81 & 2.73 & 1.46 \\
\hline 72 & 2.20 & 1.92 & 2.55 & 1.50 \\
\hline
\end{tabular}

720 


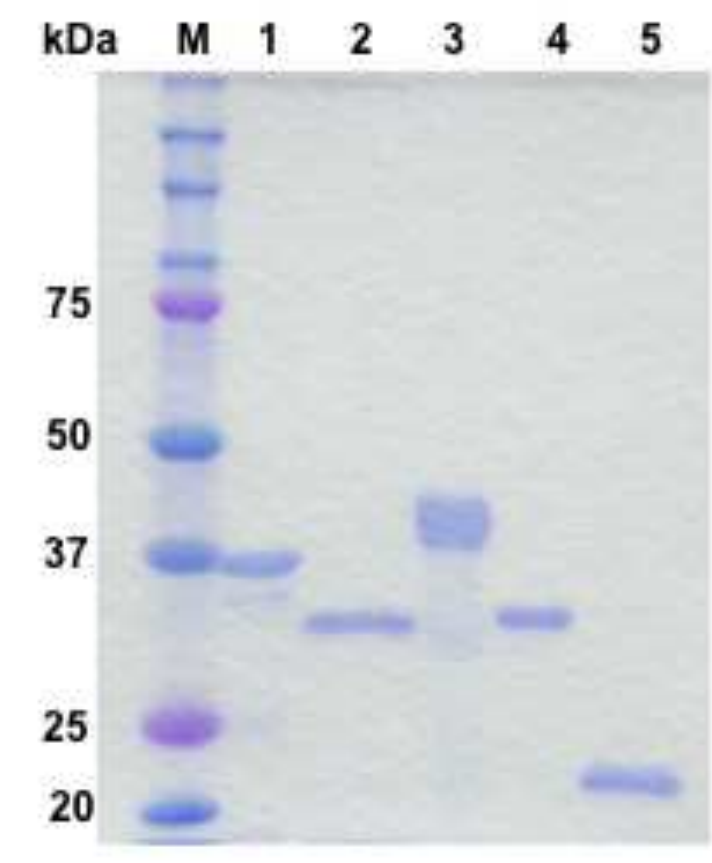




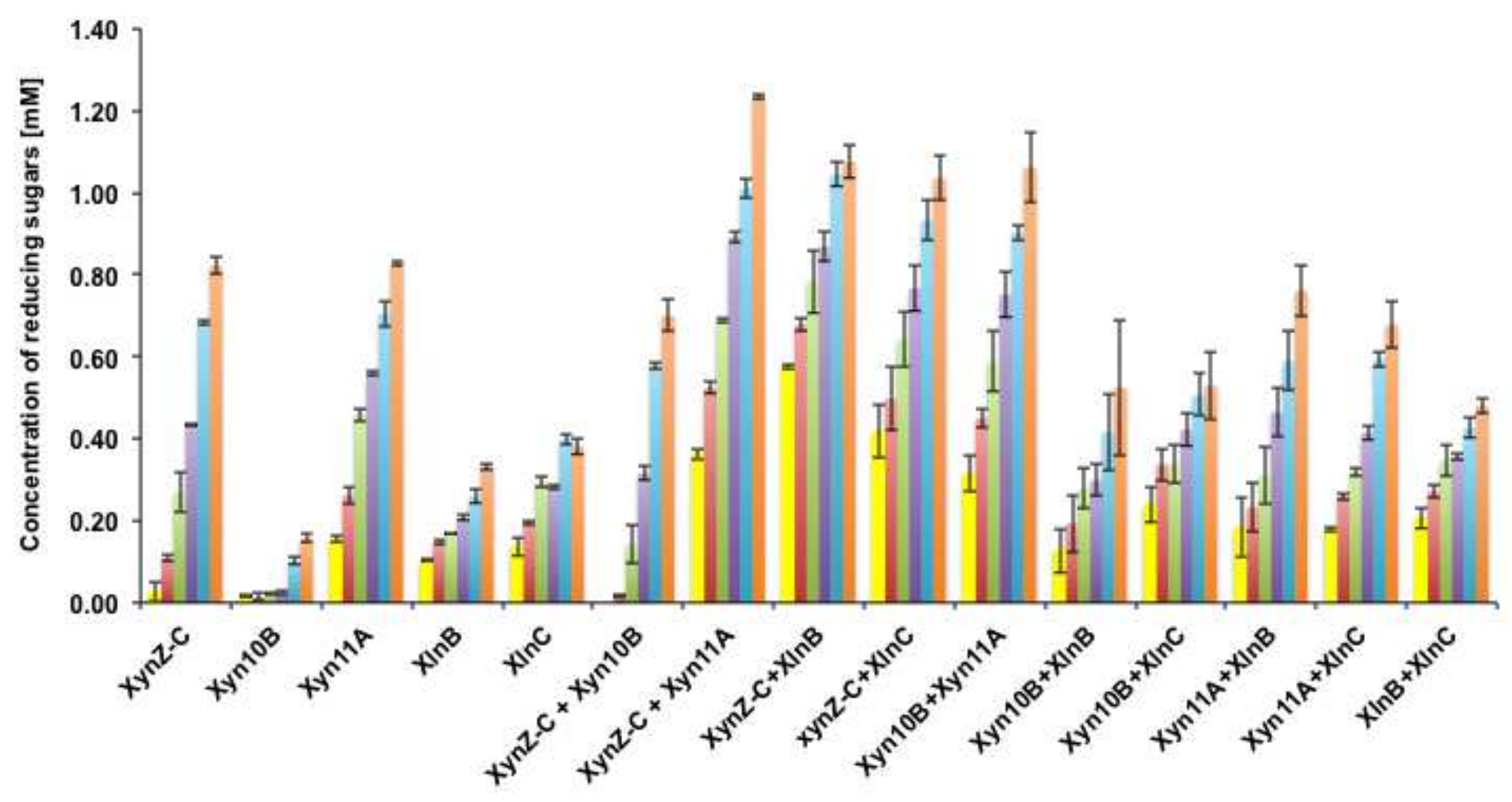




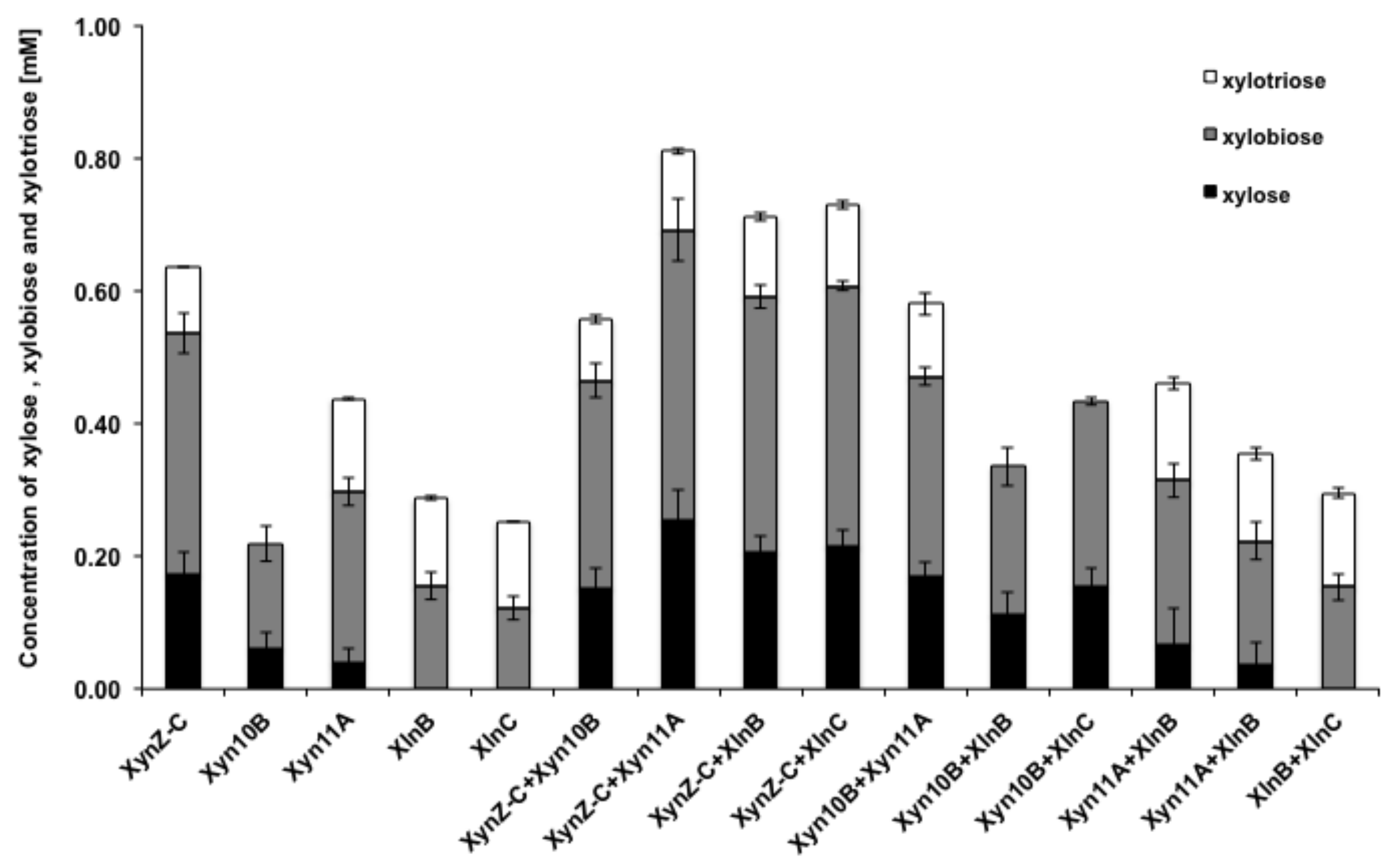




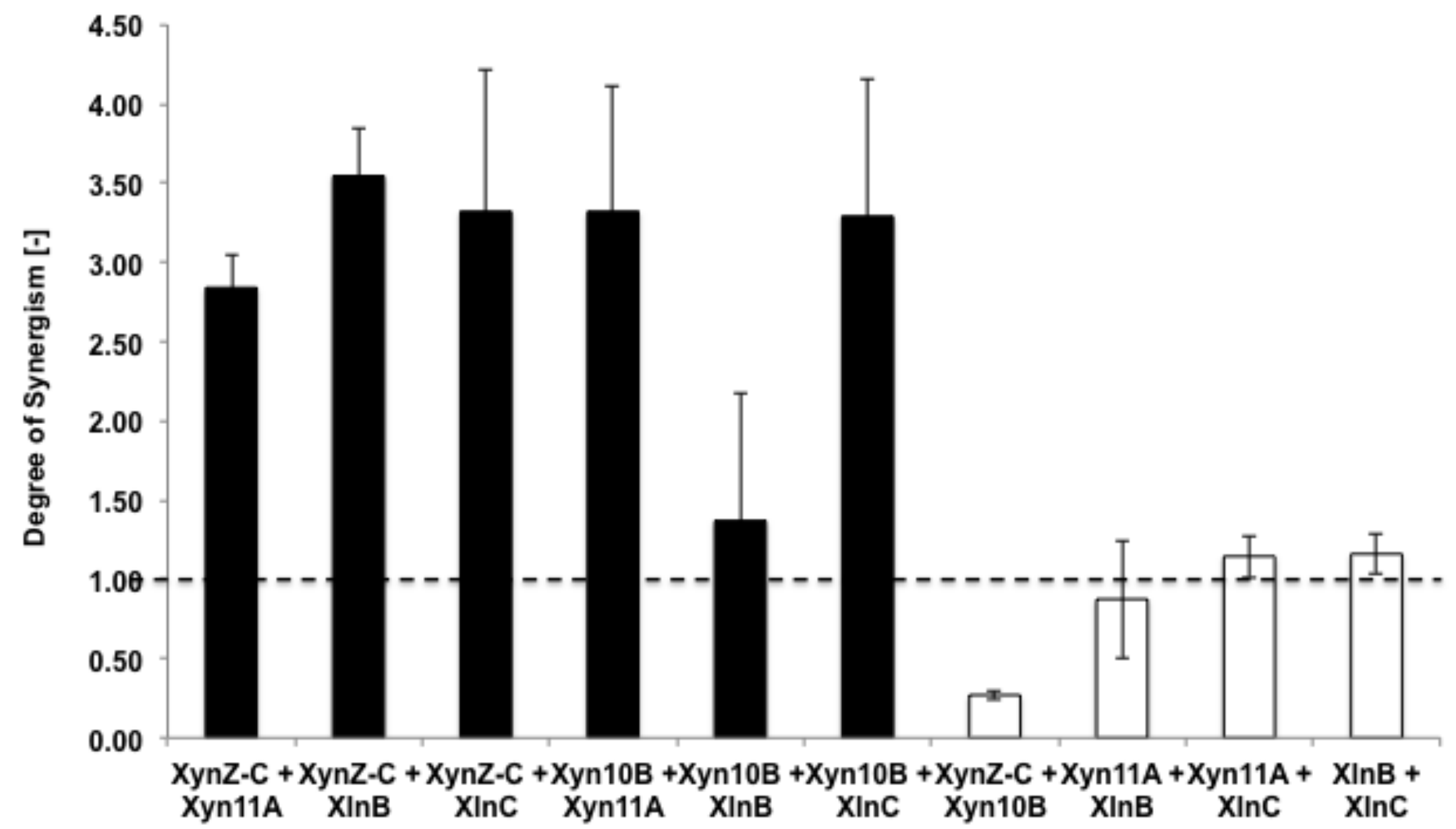




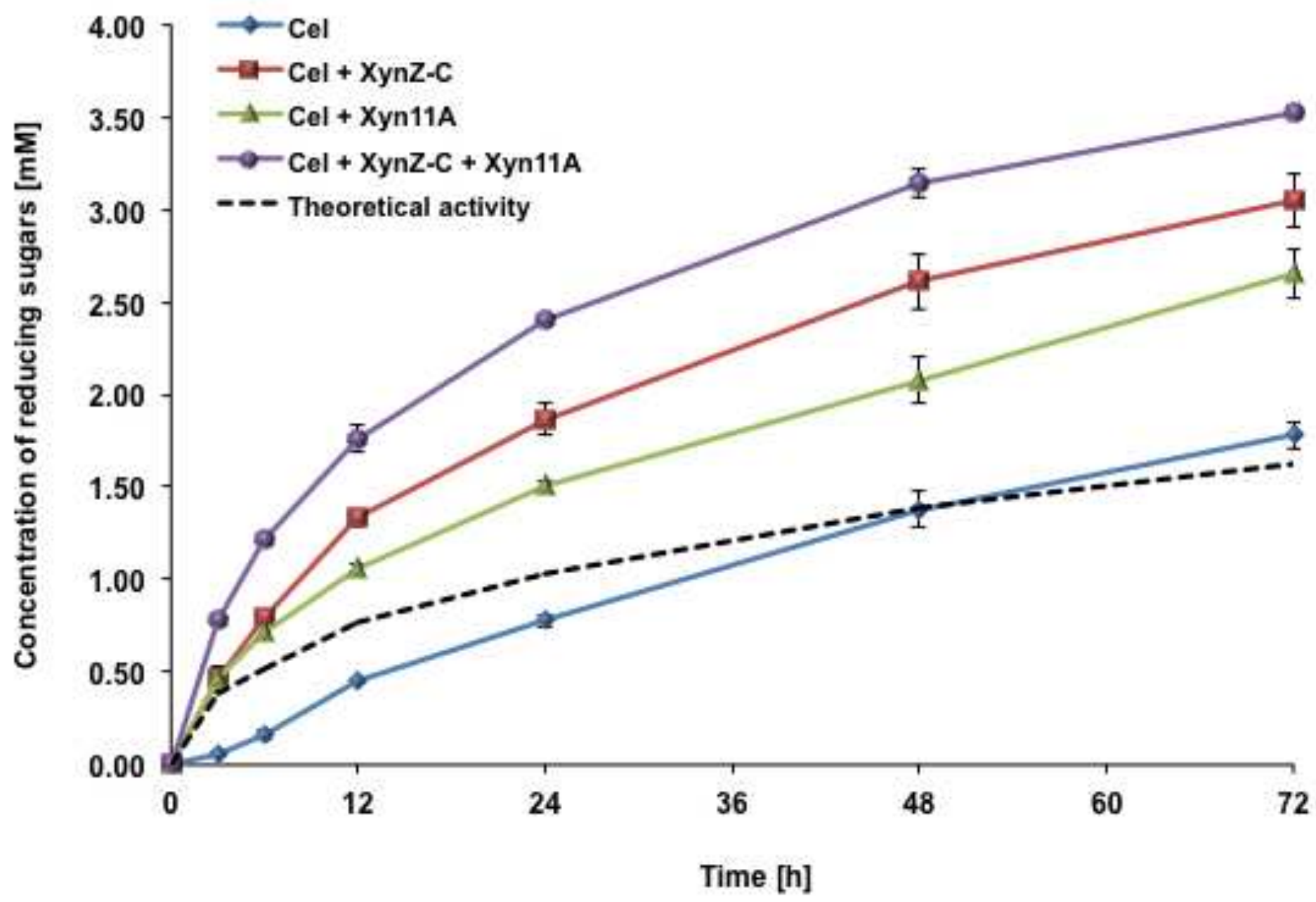

